\title{
Transaxial Plane
}

National Cancer Institute

\section{Source}

National Cancer Institute. Transaxial Plane. NCI Thesaurus. Code C94994.

Across (i.e. perpendicular to) the axial direction. When describing the plane of an image, it indicates the plane perpendicular to the long axis of the subject's body. 